\title{
Graviton propagator in a 2-parameter family of de Sitter breaking gauges
}

\author{
D. Glavan, ${ }^{a}$ S.P. Miao, ${ }^{b}$ T. Prokopec ${ }^{c}$ and R.P. Woodard ${ }^{d}$ \\ ${ }^{a}$ Centre for Cosmology, Particle Physics and Phenomenology (CP3), \\ Université catholique de Louvain, Chemin du Cyclotron 2, Louvain-la-Neuve 1348, Belgium \\ ${ }^{b}$ Department of Physics, National Cheng Kung University, \\ No. 1, University Road, Tainan City 70101, Taiwan \\ ${ }^{c}$ Institute for Theoretical Physics, Spinoza Institute 83 EMME $\Phi$ Utrecht University, \\ Postbus 80.195, Utrecht 3508 TD, The Netherlands \\ ${ }^{d}$ Department of Physics, University of Florida, \\ Gainesville, FL 32611, United States \\ E-mail: drazen.glavan@uclouvain.be, spmiao5@mail.ncku.edu.tw, \\ T.Prokopec@uu.nl, woodard@phys.ufl.edu
}

ABSTRACT: We formulate the graviton propagator on de Sitter background in a 2-parameter family of simple gauges which break de Sitter invariance. Explicit results are derived for the first order perturbations in each parameter. These results should be useful in computations to check for gauge dependence of graviton loop corrections.

KeYwords: Cosmology of Theories beyond the SM, Models of Quantum Gravity, Renormalization Regularization and Renormalons

ARXIV EPRINT: 1908.06064 


\section{Contents}

1 Introduction 1

2 A 2-parameter family of gauges 2

3 Our solution for the propagator 3

3.1 Lessons from flat space 3

3.2 Integrated propagators in de Sitter 5

$\begin{array}{lll}3.3 & \text { The } \delta \alpha \text { and } \delta \beta \text { perturbations } & 7\end{array}$

4 Discussion $\quad 8$

$\begin{array}{ll}\text { A Integrated propagators } & 10\end{array}$

$\begin{array}{ll}\text { A.1 Reflecting derivatives } & 10\end{array}$

$\begin{array}{lll}\text { A.2 Results } & 11\end{array}$

$\begin{array}{lll}\text { A.2.1 Non-integer power series } & 14\end{array}$

$\begin{array}{lll}\text { A.2.2 Integer power series } & 15\end{array}$

$\begin{array}{lll}\text { A.2.3 Final expressions } & 17\end{array}$

\section{Introduction}

Explicit computations of 1PI (one-particle-irreducible) 2-point functions on de Sitter background [1-5], and their use to quantum-correct the linearized effective field equations, provide strong evidence that inflationary gravitons modify particle kinematics [6-9] and change force laws [10]. However, there is a persistent anxiety about the reality of these effects owing to the possibility of dependence on the gauge used to define the graviton propagator. The computations mentioned above were all made in a very simple gauge that breaks de Sitter invariance $[11,12]$. When the vacuum polarization was computed in a vastly more complicated, 1-parameter family of de Sitter invariant gauges [13], the result looks very different [14], and the enhancement it provides to dynamical photons is slightly different [15], although it has the same sign and time dependence.

We seek to establish the reality of graviton corrections to particle kinematics and force laws by purging the linearized effective field equations of gauge dependence. We are developing a technique for accomplishing this by including generic parts of the quantum gravitational correlations with the source that disturbs the effective field and with the observer who measures the disturbance [16]. The technique relies on the position-space version of a set of identities derived by Donoghue [17-19] that allow one to view the infrared singular parts of an invariant amplitude as corrections to the 1PI 2-point function. We have already shown that the technique works, for graviton corrections to massless scalar 
exchange on flat space background, by making the computation in the 2-parameter family of Poincaré invariant gauges and demonstrating that the corrected 1PI 2-point function is independent of the gauge parameters [16]. Our goal is to carry out the same computation on de Sitter background, for which we require a generalization of the 2-parameter family of flat space gauges. Providing that generalization is the point of this paper.

In section 2 we review the simple gauge and propose an appropriate 2-parameter generalization. Constructing the graviton propagator as a general function of these two parameters is challenging, but almost as much information about gauge dependence can be gained by deriving just the first order variations about the simple gauge. That is done in section 3. We discuss the results in section 4. Section A consists of an appendix in which we derive explicit forms for the various integrated propagators required for the solution.

\section{A 2-parameter family of gauges}

Our de Sitter background geometry is given in conformal coordinates on $D$ spacetime dimensions with spacelike signature,

$$
d s^{2}=a^{2}\left[-d \eta^{2}+d \vec{x} \cdot d \vec{x}\right], \quad a(\eta)=-\frac{1}{H \eta}(\eta<0) .
$$

The graviton field comes from the conformally rescaled metric, $g_{\mu \nu}(x) \equiv a^{2}\left[\eta_{\mu \nu}+\kappa h_{\mu \nu}(x)\right]$. Here $\kappa^{2} \equiv 16 \pi G$ and graviton indices are raised and lowered using the Minkowski metric, $h^{\mu}{ }_{\nu} \equiv \eta^{\mu \rho} h_{\rho \nu}$.

The simple propagator is defined by the gauge fixing term [11, 12],

$$
\mathcal{L}_{\mathrm{GF}}=-\frac{1}{2} a^{D-2} \eta^{\mu \nu} F_{\mu} F_{\nu}, \quad F_{\mu}=\eta^{\rho \sigma}\left(h_{\mu \rho, \sigma}-\frac{1}{2} h_{\rho \sigma, \mu}+(D-2) H a h_{\mu \rho} \delta_{\sigma}^{0}\right) .
$$

The graviton propagator in this gauge takes the form,

$$
i\left[{ }_{\mu \nu} \Delta_{\rho \sigma}\right]\left(x ; x^{\prime}\right)=\sum_{I=A, B, C}\left[\mu \nu T_{\rho \sigma}^{I}\right] \times i \Delta_{I}\left(x ; x^{\prime}\right) .
$$

The three propagators $i \Delta_{I}\left(x ; x^{\prime}\right)$ are for minimally coupled scalars with masses $M_{A}^{2}=0$, $M_{B}^{2}=(D-2) H^{2}$ and $M_{C}^{2}=2(D-3) H^{2}$. The three tensor factors are constructed from $\eta_{\mu \nu}, \bar{\eta}_{\mu \nu} \equiv \eta_{\mu \nu}+\delta^{0}{ }_{\mu} \delta^{0}{ }_{\nu}$ and $\delta^{0}{ }_{\mu}$,

$$
\begin{aligned}
{\left[{ }_{\mu \nu} T_{\rho \sigma}^{A}\right] } & =2 \bar{\eta}_{\mu(\rho} \bar{\eta}_{\sigma) \nu}-\frac{2}{D-3} \bar{\eta}_{\mu \nu} \bar{\eta}_{\rho \sigma}, & {\left[{ }_{\mu \nu} T_{\rho \sigma}^{B}\right] } & =-4 \delta^{0}{ }_{(\mu} \bar{\eta}_{\nu)\left(\rho{ }_{\sigma)}^{0},\right.}, \\
{\left[{ }_{\mu \nu} T_{\rho \sigma}^{C}\right] } & =\frac{2 E_{\mu \nu} E_{\rho \sigma}}{(D-2)(D-3)}, & E_{\mu \nu} & \equiv(D-3) \delta^{0}{ }_{\mu} \delta^{0}{ }_{\nu}+\bar{\eta}_{\mu \nu},
\end{aligned}
$$

where parenthesized indices are symmetrized. The simple graviton propagator (2.3) is easy to use for three reasons:

1. In $D=4$ its three propagators consist of just one or two terms involving the two scale factors and the invariant interval of flat space,

$$
i \Delta_{A} \longrightarrow \frac{1}{4 \pi^{2}}\left[\frac{1}{a a^{\prime} \Delta x^{2}}-\frac{H^{2}}{2} \ln \left(H^{2} \Delta x^{2}\right)\right], \quad i \Delta_{B, C} \longrightarrow \frac{1}{4 \pi^{2} a a^{\prime} \Delta x^{2}} ;
$$


2. Its tensor factors are constants; and

3. Its 1PI 2-point functions are elementary functions of $\Delta x^{2}, a$ and $a^{\prime}$.

None of these features pertains for de Sitter invariant gauges $[13,14]$, which is why only a single loop computation has been made using them.

It is desirable to construct the graviton propagator in a 2-parameter family of de Sitter breaking gauges that can be seen as perturbations of the simple gauge [11, 12]. This also permits a 2-parameter family of gauges rather than the 1-parameter family that would be available without de Sitter breaking, owing to a topological obstacle which precludes adding de Sitter invariant gauge fixing functionals [20]. Finally, it is advantageous that the flat space limit of our gauge condition should agree with the 2-parameter family of gauges used for the flat space computation [16] which we seek to generalize to de Sitter. A plausible generalization of $(2.2)$ is therefore,

$$
\mathcal{L}_{\mathrm{GF}}^{\alpha \beta}=-\frac{a^{D-2}}{2 \alpha} \eta^{\mu \nu} \mathcal{F}_{\mu} \mathcal{F}_{\nu}, \quad \mathcal{F}_{\mu}=\eta^{\rho \sigma}\left(h_{\mu \rho, \sigma}-\frac{\beta}{2} h_{\rho \sigma, \mu}+(D-2) H a h_{\mu \rho} \delta_{\sigma}^{0}\right) .
$$

Note that taking $\alpha=\beta=1$ corresponds to the simple gauge (2.2), and that taking the flat space limit ( $H=0$ and $a=1)$ recovers the family of gauges in which the flat space calculation [16] was made.

\section{Our solution for the propagator}

The purpose of this section is to construct the first order perturbations in $\delta \alpha \equiv \alpha-1$ and $\delta \beta \equiv \beta-1$ of the propagator in the gauge (2.7). We first review the flat space propagator, and its use in studies of gauge dependence [16], to make two points:

1. The result will involve convolutions of propagators; and

2. First order perturbations in the gauge parameters provide almost as much information about gauge as the all-orders result.

We then return to de Sitter to define the necessary integrated propagators, whose evaluation is consigned to the appendix. The section closes with results for the first order perturbations in $\delta \alpha$ and $\delta \beta$.

\subsection{Lessons from flat space}

In the flat space limit of (2.7) the graviton propagator is [21],

$$
\begin{aligned}
i\left[{ }_{\mu \nu} \Delta_{\rho \sigma}^{\mathrm{flat}}\right]\left(x ; x^{\prime}\right)= & \left\{2 \Pi_{\mu(\rho} \Pi_{\sigma) \nu}-\frac{2}{D-1} \Pi_{\mu \nu} \Pi_{\rho \sigma}\right. \\
& -\frac{2}{(D-2)(D-1)}\left[\eta_{\mu \nu}-\left(\frac{D \beta-2}{\beta-2}\right) \frac{\partial_{\mu} \partial_{\nu}}{\partial^{2}}\right]\left[\eta_{\rho \sigma}-\left(\frac{D \beta-2}{\beta-2}\right) \frac{\partial_{\rho} \partial_{\sigma}}{\partial^{2}}\right] \\
& \left.+4 \alpha \times \frac{\partial_{(\mu} \Pi_{\nu)(\rho} \partial_{\sigma)}}{\partial^{2}}+\frac{4 \alpha}{(\beta-2)^{2}} \times \frac{\partial_{\mu} \partial_{\nu} \partial_{\rho} \partial_{\sigma}}{\partial^{4}}\right\} i \Delta\left(x ; x^{\prime}\right) .
\end{aligned}
$$


Here the transverse projection operator is $\Pi_{\mu \nu} \equiv \eta_{\mu \nu}-\frac{\partial_{\mu} \partial_{\nu}}{\partial^{2}}$ and the massless scalar propagator in flat space is,

$$
i \Delta\left(x ; x^{\prime}\right)=\frac{\Gamma\left(\frac{D}{2}-1\right)}{4 \pi^{\frac{D}{2}} \Delta x^{D-2}} \quad \Longrightarrow \quad \partial^{2} i \Delta\left(x ; x^{\prime}\right)=i \delta^{D}\left(x-x^{\prime}\right) .
$$

First note from the inverse powers of $\partial^{2}$ which act on $i \Delta\left(x ; x^{\prime}\right)$ that the general gauge propagator (3.1) involves the convolution of one propagator with another [22], ${ }^{1}$

$$
\frac{1}{\partial^{2}} i \Delta\left(x ; x^{\prime}\right)=-i \int d^{D} z i \Delta(x ; z) i \Delta\left(z ; x^{\prime}\right)=\frac{\Gamma\left(\frac{D}{2}-2\right)}{16 \pi^{\frac{D}{2}}}\left[\mu^{D-4}-\frac{1}{\Delta x^{D-4}}\right]
$$

where $\mu$ is a regularization scale. The flat space limit therefore implies that similar convolutions must occur in the general gauge propagator on de Sitter background.

Now expand the gauge parameters around their "simple" values,

$$
\alpha \equiv 1+\delta \alpha, \quad \beta \equiv 1+\delta \beta,
$$

Expanding the general gauge propagator (3.1) in $\delta \alpha$ and $\delta \beta$ gives,

$$
\begin{aligned}
i\left[{ }_{\mu \nu} \Delta_{\rho \sigma}^{\mathrm{flat}}\right]= & {\left[2 \eta_{\mu(\rho} \eta_{\sigma) \nu}-\frac{2 \eta_{\mu \nu} \eta_{\rho \sigma}}{D-2}\right] i \Delta\left(x ; x^{\prime}\right)+\delta \alpha \times\left[\frac{4 \partial_{(\mu} \eta_{\nu)(\rho} \partial_{\sigma)}}{\partial^{2}}\right] i \Delta\left(x ; x^{\prime}\right) } \\
& -\delta \beta \times \frac{4}{D-2}\left[\frac{\eta_{\mu \nu} \partial_{\rho} \partial_{\sigma}}{\partial^{2}}+\frac{\partial_{\mu} \partial_{\nu} \eta_{\rho \sigma}}{\partial^{2}}\right] i \Delta\left(x ; x^{\prime}\right)+O\left(\delta \alpha \delta \beta, \delta \beta^{2}\right) .
\end{aligned}
$$

Table 1 concerns gauge dependence in one graviton loop corrections to the effective field equation for a massless, minimally coupled scalar on flat space background [16]. The highly gauge dependent contribution from the 1PI 2-point function is $i=0$, and the five source and observer corrections are reported, both for the full propagator (3.1) on the left, and for the first three terms in the expansion (3.5) on the right. The left hand table follows the cancellation of three distinct combinations of the gauge parameters: $\alpha, \frac{1}{\beta-2}$ and $\frac{(\alpha-3)}{(\beta-2)^{2}}$. The right hand table follows two: $\delta \alpha$ and $\delta \beta$. So the vastly simpler first order perturbations (3.5) provide two thirds of the checks available from the full propagator (3.1).

\footnotetext{
${ }^{1}$ The $\Delta x$-dependent part of (3.2) follows from the usual procedure of combining denominators, shifting, Wick-rotating and evaluating the Euclidean-space integration,

$$
\begin{aligned}
-i \int & d^{D} z \frac{\Gamma\left(\frac{D}{2}-1\right)}{4 \pi^{\frac{D}{2}}}\left[\frac{1}{(x-z)^{2}+i \epsilon}\right]^{\frac{D}{2}-1} \times \frac{\Gamma\left(\frac{D}{2}-1\right)}{4 \pi^{\frac{D}{2}}}\left[\frac{1}{\left(z-x^{\prime}\right)^{2}+i \epsilon}\right]^{\frac{D}{2}-1} \\
& =-\frac{i \Gamma(D-2)}{16 \pi^{D}} \int_{0}^{1} d s s^{\frac{D}{2}-2}(1-s)^{\frac{D}{2}-2} \int \frac{d^{D} z}{\left[s(x-z)^{2}+(1-s)\left(z-x^{\prime}\right)^{2}+i \epsilon\right]^{D-2}} \\
& =-\frac{\Gamma(D-2)}{16 \pi^{D}} \int_{0}^{1} d s s^{\frac{D}{2}-2}(1-s)^{\frac{D}{2}-2} \int \frac{d^{D} z_{E}}{\left[z_{E}^{2}+s(1-s) \Delta x^{2}\right]^{D-2}}, \\
& =-\frac{\Gamma(D-2)}{16 \pi^{D}} \int_{0}^{1} d s s^{\frac{D}{2}-2}(1-s)^{\frac{D}{2}-2} \times \frac{\pi^{\frac{D}{2}}}{\Gamma\left(\frac{D}{2}\right)\left[s(1-s) \Delta x^{2}\right]^{\frac{D}{2}-2}} \times \frac{\Gamma\left(\frac{D}{2}-2\right) \Gamma\left(\frac{D}{2}\right)}{\Gamma(D-2)}
\end{aligned}
$$
}




\begin{tabular}{|c|c|c|c|c|}
\hline$i$ & 1 & $\alpha$ & $\frac{1}{\beta-2}$ & $\frac{(\alpha-3)}{(\beta-2)^{2}}$ \\
\hline 0 & $+\frac{3}{4}$ & $-\frac{3}{4}$ & $-\frac{3}{2}$ & $+\frac{3}{4}$ \\
\hline 1 & 0 & 0 & 0 & +1 \\
\hline 2 & 0 & 0 & 0 & 0 \\
\hline 3 & 0 & 0 & +3 & -2 \\
\hline 4 & $+\frac{17}{4}$ & $-\frac{3}{4}$ & 0 & $-\frac{1}{4}$ \\
\hline 5 & -2 & $+\frac{3}{2}$ & $-\frac{3}{2}$ & $+\frac{1}{2}$ \\
\hline Total & +3 & 0 & 0 & 0 \\
\hline
\end{tabular}

\begin{tabular}{|c|c|c|c|}
\hline$i$ & 1 & $\delta \alpha$ & $\delta \beta$ \\
\hline 0 & 0 & 0 & $-\frac{3}{2}$ \\
\hline 1 & -2 & +1 & -4 \\
\hline 2 & 0 & 0 & 0 \\
\hline 3 & +1 & -2 & +5 \\
\hline 4 & +4 & -1 & +1 \\
\hline 5 & 0 & +2 & $-\frac{1}{2}$ \\
\hline Total & +3 & 0 & 0 \\
\hline
\end{tabular}

Table 1. The left hand table gives the various gauge-dependent multiplicative factors for one graviton corrections to the massless scalar exchange force on flat space background using the full propagator (3.1) on the left, and for the first order expansion (3.5) on the right. The contribution of the 1PI 2-point function is $i=0$ and the other values of $i$ correspond to different source and observer corrections that are precisely defined in [16] and which are necessary to eliminate gauge dependence.

\subsection{Integrated propagators in de Sitter}

The insights we have just derived from the flat space limit motivate constructing just the order $\delta \alpha$ and $\delta \beta$ perturbations of the (2.7) propagator,

$$
i\left[{ }_{\mu \nu} \Delta_{\rho \sigma}\right]=i\left[{ }_{\mu \nu} \Delta_{\rho \sigma}^{0}\right]+\delta \alpha \times i\left[{ }_{\mu \nu} \Delta_{\rho \sigma}^{\alpha}\right]+\delta \beta \times i\left[{ }_{\mu \nu} \Delta_{\rho \sigma}^{\beta}\right]+O\left(\delta^{2}\right)
$$

The flat space limit also implies that these perturbations must involve integrated propagators analogous to (3.3). To understand more fully what these are, recall that the 0 -th order propagator $i\left[{ }_{\mu \nu} \Delta_{\rho \sigma}^{0}\right]\left(x ; x^{\prime}\right)$ takes the form $(2.3)$ where the tensor factors $(2.4)-(2.5)$ represent a sort of $3+1$ decomposition into purely spatial ( $A$-type), mixed space and time ( $B$-type), and temporal plus trace ( $C$-type) terms. The corresponding $A, B$ and $C$ scalar propagators are most easily represented in terms of the de Sitter length function $y\left(x ; x^{\prime}\right)$,

$$
y\left(x ; x^{\prime}\right) \equiv H^{2} a(\eta) a\left(\eta^{\prime}\right)\left[\left\|\vec{x}-\vec{x}^{\prime}\right\|^{2}-\left(\left|\eta-\eta^{\prime}\right|-i \epsilon\right)^{2}\right] .
$$

The $A$-type propagator breaks de Sitter invariance [23, 24],

$$
\begin{aligned}
i \Delta_{A}= & \frac{H^{D-2}}{(4 \pi)^{\frac{D}{2}}}\left\{\frac{\Gamma\left(\frac{D}{2}\right)}{\frac{D}{2}-1}\left(\frac{4}{y}\right)^{\frac{D}{2}-1}+\frac{\Gamma\left(\frac{D}{2}+1\right)}{\frac{D}{2}-2}\left(\frac{4}{y}\right)^{\frac{D}{2}-2}-\frac{\Gamma(D-1)}{\Gamma\left(\frac{D}{2}\right)}\left[\pi \cot \left(\pi \frac{D}{2}\right)-\ln \left(a a^{\prime}\right)\right]\right. \\
& \left.+\sum_{n=1}^{\infty}\left[\frac{1}{n} \frac{\Gamma(n+D-1)}{\Gamma\left(n+\frac{D}{2}\right)}\left(\frac{y}{4}\right)^{n}-\frac{1}{n-\frac{D}{2}+2} \frac{\Gamma\left(n+\frac{D}{2}+1\right)}{\Gamma(n+2)}\left(\frac{y}{4}\right)^{n-\frac{D}{2}+2}\right]\right\}
\end{aligned}
$$


In contrast, the $B$-type and $C$-type propagators are de Sitter invariant, ${ }^{2}$

$$
\begin{gathered}
i \Delta_{B}=\frac{H^{D-2}}{(4 \pi)^{\frac{D}{2}}}\left\{\frac{\Gamma\left(\frac{D}{2}\right)}{\frac{D}{2}-1}\left(\frac{4}{y}\right)^{\frac{D}{2}-1}+\sum_{n=0}^{\infty}\left[\frac{\Gamma\left(n+\frac{D}{2}\right)}{\Gamma(n+2)}\left(\frac{y}{4}\right)^{n-\frac{D}{2}+2}-\frac{\Gamma(n+D-2)}{\Gamma\left(n+\frac{D}{2}\right)}\left(\frac{y}{4}\right)^{n}\right]\right\}, \\
i \Delta_{C}=\frac{H^{D-2}}{(4 \pi)^{\frac{D}{2}}}\left\{\frac{\Gamma\left(\frac{D}{2}\right)}{\frac{D}{2}-1}\left(\frac{4}{y}\right)^{\frac{D}{2}-1}-\sum_{n=0}^{\infty}\left[\left(n-\frac{D}{2}+3\right) \frac{\Gamma\left(n+\frac{D}{2}-1\right)}{\Gamma(n+2)}\left(\frac{y}{4}\right)^{n-\frac{D}{2}+2}\right.\right. \\
\left.\left.-(n+1) \frac{\Gamma(n+D-3)}{\Gamma\left(n+\frac{D}{2}\right)}\left(\frac{y}{4}\right)^{n}\right]\right\} .
\end{gathered}
$$

We obviously require the convolution of any pair of these three propagators. There is also the matter of derivatives, and the crucial factors of $1 / a$ with which they are associated on de Sitter. Although the integrated propagators of flat space (3.5) always carry two derivatives $\frac{\partial_{\mu} \partial_{\nu}}{\partial^{2}} \times i \Delta\left(x ; x^{\prime}\right)$, those on de Sitter can also have one derivative or none. Hence our integrated propagators on de Sitter involve a measure factor of $a^{D}$, divided by zero, one, or two powers of $a$,

$$
\begin{aligned}
I_{\mu \nu}\left(x ; x^{\prime}\right) & \equiv-i \int d^{D} z a_{z}^{D} i \Delta_{\mu}(x ; z) i \Delta_{\nu}\left(z ; x^{\prime}\right), \\
J_{\mu \nu}\left(x ; x^{\prime}\right) & \equiv-i \int d^{D} z a_{z}^{D-1} i \Delta_{\mu}(x ; z) i \Delta_{\nu}\left(z ; x^{\prime}\right), \\
K_{\mu \nu}\left(x ; x^{\prime}\right) & \equiv-i \int d^{D} z a_{z}^{D-2} i \Delta_{\mu}(x ; z) i \Delta_{\nu}\left(z ; x^{\prime}\right) .
\end{aligned}
$$

The appendix derives explicit results for these expressions.

As explained, the $I_{\mu \nu}\left(x ; x^{\prime}\right)$ integrals carry no derivatives, the $J_{\mu \nu}\left(x ; x^{\prime}\right)$ carry one derivative, and the $K_{\mu \nu}\left(x ; x^{\prime}\right)$ integrals carry two derivatives. Although these derivatives could be reflected outside the $z^{\mu}$ integration to act on either of the external variables $x^{\mu}$ or $x^{\prime \mu}$, the result is cumbersome. (The procedure is explained in the appendix.) It is also possible that the effort would be wasted if reducing the diagram in which the perturbed propagator resides would be facilitated by retaining the original derivatives, or by reflecting them to the opposite coordinate. We have therefore devised a notation in which the symbol " $D_{\mu}$ " that $\frac{\partial}{\partial z^{\mu}}$ acts on the left hand propagator. The notation " $\mathcal{D}_{\mu}$ " indicates that $\frac{\partial}{\partial z^{\mu}}$ acts on the right hand propagator. Some examples are,

$$
\begin{aligned}
D_{\mu} J_{\rho \sigma}\left(x ; x^{\prime}\right) & \equiv-i \int d^{D} z a_{z}^{D-1} \frac{\partial i \Delta_{\rho}(x ; z)}{\partial z^{\mu}} i \Delta_{\sigma}\left(z ; x^{\prime}\right), \\
\mathcal{D}_{\mu} J_{\rho \sigma}\left(x ; x^{\prime}\right) & \equiv-i \int d^{D} z a_{z}^{D-1} i \Delta_{\rho}(x ; z) \frac{\partial i \Delta_{\sigma}\left(z ; x^{\prime}\right)}{\partial z^{\mu}}, \\
D_{\mu} D_{\nu} K_{\rho \sigma}\left(x ; x^{\prime}\right) & \equiv-i \int d^{D} z a_{z}^{D-2} \frac{\partial^{2} i \Delta_{\mu}(x ; z)}{\partial z^{\mu} \partial z^{\nu}} i \Delta_{\nu}\left(z ; x^{\prime}\right) .
\end{aligned}
$$

\footnotetext{
${ }^{2}$ The infinite sums (3.8)-(3.10) might seem intimidating but the simple $D=4$ limits (2.6) mean that only a few of the lowest terms need to be retained when divergences are present.
} 


\subsection{The $\delta \alpha$ and $\delta \beta$ perturbations}

Suppose we invert a full kinetic operator $\mathbf{D}$ which can be expressed as the sum of a 0 -th order $\mathbf{D}_{0}$ operator and a perturbation $\mathbf{D}_{1}$. The full propagator can be expanded in familiar geometric series,

$$
\frac{i}{\mathbf{D}_{0}+\mathbf{D}_{1}}=\frac{i}{\mathbf{D}_{0}}+\frac{i}{\mathbf{D}_{0}} \times i \mathbf{D}_{1} \times \frac{i}{\mathbf{D}_{0}}+\frac{i}{\mathbf{D}_{0}} \times i \mathbf{D}_{1} \times \frac{i}{\mathbf{D}_{0}} \times i \mathbf{D}_{1} \times \frac{i}{\mathbf{D}_{0}}+\ldots
$$

The first order perturbation is the second term on the right hand side of (3.17).

Let us define the kinetic operator of a massless, minimally coupled scalar as $D_{A} \equiv$ $\partial_{\mu} a^{D-2} \partial^{\mu}$. The graviton kinetic operator in our gauge (2.7) is,

$$
\begin{aligned}
\mathcal{D}^{\mu \nu \rho \sigma}= & \frac{1}{2}\left[\eta^{\mu(\rho} \eta^{\sigma) \nu}-\left(\frac{2 \alpha-\beta^{2}}{2 \alpha}\right) \eta^{\mu \nu} \eta^{\rho \sigma}\right] D_{A}+\left(\frac{D-2}{\alpha}\right) H^{2} a^{D} \delta_{0}^{(\mu} \eta^{\nu)(\rho} \delta_{0}^{\sigma)} \\
& -\left(\frac{\alpha-1}{\alpha}\right) \partial^{(\rho} a^{D-2} \eta^{\sigma)(\mu} \partial^{\nu)}+\left(\frac{\alpha-\beta}{2 \alpha}\right)\left[a^{D-2} \partial^{\mu} \partial^{\nu} \eta^{\rho \sigma}+\eta^{\mu \nu} \partial^{\rho} \partial^{\sigma} a^{D-2}\right] .
\end{aligned}
$$

Expanding the kinetic operator (3.18) in $\delta \alpha$ and $\delta \beta$ gives,

$$
\mathcal{D}^{\mu \nu \rho \sigma}=\mathcal{D}_{0}^{\mu \nu \rho \sigma}+\delta \alpha \times \mathcal{D}_{\alpha}^{\mu \nu \rho \sigma}+\delta \beta \times \mathcal{D}_{\beta}^{\mu \nu \rho \sigma}+O\left(\delta^{2}\right),
$$

where the first three operators are,

$$
\begin{aligned}
\mathcal{D}_{0}^{\mu \nu \rho \sigma}= & \frac{1}{2} \eta^{\mu(\rho} \eta^{\sigma) \nu} D_{A}-\frac{1}{4} \eta^{\mu \nu} \eta^{\rho \sigma} D_{A}+(D-2) H^{2} a^{D} \delta_{0}^{(\mu} \eta^{\nu)(\rho} \delta_{0}^{\sigma)}, \\
\mathcal{D}_{\alpha}^{\mu \nu \rho \sigma}=-\frac{1}{4} \eta^{\mu \nu} \eta^{\rho \sigma} D_{A}-(D-2) H^{2} a^{D} \delta^{(\mu}{ }_{0} \eta^{\nu)(\rho} \delta^{\sigma)}{ }_{0} & -\partial^{(\rho} a^{D-2} \eta^{\sigma)(\mu} \partial^{\nu)}+\frac{1}{2}\left[a^{D-2} \partial^{\mu} \partial^{\nu} \eta^{\rho \sigma}+\eta^{\mu \nu} \partial^{\rho} \partial^{\sigma} a^{D-2}\right], \\
\mathcal{D}_{\beta}^{\mu \nu \rho \sigma}= & \frac{1}{2} \eta^{\mu \nu} \eta^{\rho \sigma} D_{A}-\frac{1}{2}\left[a^{D-2} \partial^{\mu} \partial^{\nu} \eta^{\rho \sigma}+\eta^{\mu \nu} \partial^{\rho} \partial^{\sigma} a^{D-2}\right]
\end{aligned}
$$

From expression (3.17) the $\delta \alpha$ perturbation of the propagator is,

$$
\left.i\left[{ }_{\mu \nu} \Delta_{\rho \sigma}^{\alpha}\right]\left(x ; x^{\prime}\right)=\int d^{D} z i\left[{ }_{\mu \nu} \Delta_{\alpha \beta}^{0}\right](x ; z) \times i \mathcal{D}_{\alpha}^{\alpha \beta \gamma \delta} \times i{ }_{\gamma \delta} \Delta_{\rho \sigma}^{0}\right]\left(z ; x^{\prime}\right) .
$$

We next substitute expressions (2.3) for the 0 -th order propagator, and (3.21) for the $\delta \alpha$ perturbation of the kinetic operator. After some tedious manipulations the result can be expressed in terms of the integrated propagators (3.11)-(3.13),

$$
\begin{aligned}
\left.i{ }_{\mu \nu} \Delta_{\rho \sigma}^{\alpha}\right]= & 4(D-2) H^{2} \delta^{0}{ }_{(\mu} \bar{\eta}_{\nu)(\rho} \delta_{\sigma)}^{0} I_{B B}-\frac{4 H^{2}}{D-2} E_{\mu \nu} E_{\rho \sigma} I_{C C} \\
& +\frac{4 H}{D-3}\left\{-(D-2) \bar{\eta}_{\mu \nu} \delta^{0}{ }_{(\rho} \bar{D}_{\sigma)} J_{A B}-\bar{\eta}_{\mu \nu} E_{\rho \sigma} D_{0} J_{A C}+E_{\mu \nu} \delta_{(\rho}^{0} \bar{D}_{\sigma)} J_{C B}\right. \\
& \left.+\frac{E_{\mu \nu} E_{\rho \sigma}}{D-2} D_{0} J_{C C}\right\}+\frac{4 H}{D-3}\left\{-(D-2) \bar{\eta}_{\rho \sigma} \delta_{(\mu}^{0} \overline{\mathcal{D}}_{\nu)} J_{B A}-E_{\mu \nu} \bar{\eta}_{\rho \sigma} \mathcal{D}_{0} J_{C A}\right.
\end{aligned}
$$




$$
\begin{aligned}
& \left.+\delta_{(\mu}^{0} \overline{\mathcal{D}}_{\nu)} E_{\rho \sigma} J_{B C}+\frac{E_{\mu \nu} E_{\rho \sigma}}{D-2} \mathcal{D}_{0} J_{C C}\right\}+\frac{4 D_{0} \mathcal{D}_{0}}{(D-3)^{2}}\left\{\bar{\eta}_{\mu \nu} \bar{\eta}_{\rho \sigma} K_{A A}+E_{\mu \nu} E_{\rho \sigma} K_{C C}\right. \\
& \left.-\bar{\eta}_{\mu \nu} E_{\rho \sigma} K_{A C}-E_{\mu \nu} \bar{\eta}_{\rho \sigma} K_{C A}\right\}+\frac{4}{D-3}\left\{\delta_{(\rho}^{0} \bar{D}_{\sigma)} \mathcal{D}_{0}\left[-\bar{\eta}_{\mu \nu} K_{A B}+E_{\mu \nu} K_{C B}\right]\right. \\
& \left.-D_{0} \delta_{(\mu}^{0} \overline{\mathcal{D}}_{\nu)}\left[\bar{\eta}_{\rho \sigma} K_{B A}-E_{\rho \sigma} K_{B C}\right]\right\}-4\left\{\bar{D}_{(\rho} \bar{\eta}_{\sigma)(\mu} \overline{\mathcal{D}}_{\nu)} K_{A A}+\delta_{(\mu}^{0} \bar{\eta}_{\nu)(\rho)} \delta_{\sigma)}^{0} D_{0} \mathcal{D}_{0}\right. \\
& \left.\times K_{B B}-\delta_{(\rho}^{0} \bar{D}_{\sigma)} \delta_{(\mu}^{0} \overline{\mathcal{D}}_{\nu)} K_{B B}+D_{0} \overline{\mathcal{D}}_{(\mu} \bar{\eta}_{\nu)(\rho)} \delta_{\sigma)}^{0} K_{A B}+\bar{D}_{(\rho} \bar{\eta}_{\sigma)(\mu} \delta_{\nu)}^{0} \mathcal{D}_{0} K_{B A}\right\} .
\end{aligned}
$$

Recall that $\bar{\eta}^{\mu \nu}=\eta^{\mu \nu}+\delta_{0}^{\mu} \delta^{\nu}$ and $E^{\mu \nu} \equiv(D-3) \delta_{0}^{\mu} \delta_{0}^{\nu}+\bar{\eta}^{\mu \nu}$.

By analogy with (3.23), the $\delta \beta$ perturbation is,

$$
\left.i\left[{ }_{\mu \nu} \Delta_{\rho \sigma}^{\beta}\right]\left(x ; x^{\prime}\right)=\int d^{D} z i\left[{ }_{\mu \nu} \Delta_{\alpha \beta}^{0}\right](x ; z) \times i \mathcal{D}_{\beta}^{\alpha \beta \gamma \delta} \times i{ }_{\gamma \delta} \Delta_{\rho \sigma}^{0}\right]\left(z ; x^{\prime}\right) .
$$

Substituting the 0 -th order propagator (2.3) and the $\delta \beta$ perturbation of the knietic operator (3.22) gives,

$$
\begin{aligned}
\left.i{ }_{\mu \nu} \Delta_{\rho \sigma}^{\beta}\right]= & -\frac{4(D-1) H^{2}}{(D-3)^{2}}\left\{(D-2) \bar{\eta}_{\mu \nu} \bar{\eta}_{\rho \sigma} I_{A A}-\left[\bar{\eta}_{\mu \nu} E_{\rho \sigma}+E_{\mu \nu} \bar{\eta}_{\rho \sigma}\right] I_{A C}\right. \\
& \left.+\frac{E_{\mu \nu} E_{\rho \sigma} I_{C C}}{D-2}\right\}-\frac{4 \bar{\eta}_{\mu \nu}}{D-3}\left\{\bar{D}_{\rho} \bar{D}_{\sigma} K_{A A}+2 \delta^{0}{ }_{(\rho} \bar{D}_{\sigma)} D_{0} K_{A B}+\delta_{\rho}^{0} \delta^{0}{ }_{\sigma} D_{0}^{2} K_{A C}\right. \\
& \left.+\frac{\bar{\eta}_{\rho \sigma} D_{0}^{2}\left(K_{A C}-K_{A A}\right)}{D-3}\right\}+\frac{4 E_{\mu \nu}}{(D-3)(D-2)}\left\{\bar{D}_{\rho} \bar{D}_{\sigma} K_{C A}+2 \delta^{0}{ }_{(\rho} \bar{D}_{\sigma)} D_{0} K_{C B}\right. \\
& \left.+\delta^{0} \delta^{\delta^{0}}{ }_{\sigma} D_{0}^{2} K_{C C}+\frac{\bar{\eta}_{\rho \sigma} D_{0}^{2}\left(K_{C C}-K_{C A}\right)}{D-3}\right\}-\frac{4 \bar{\eta}_{\rho \sigma}}{D-3}\left\{\overline{\mathcal{D}}_{\mu} \overline{\mathcal{D}}_{\nu} K_{A A}\right. \\
& \left.+2 \delta^{0}{ }_{(\mu} \overline{\mathcal{D}}_{\nu} \mathcal{D}_{0} K_{B A}+\delta_{\mu}^{0} \delta^{0}{ }_{\nu} \mathcal{D}_{0}{ }^{2} K_{C A}+\frac{\bar{\eta}_{\mu \nu} \mathcal{D}_{0}{ }^{2}\left(K_{C A}-K_{A A}\right)}{D-3}\right\}+\frac{4 E_{\rho \sigma}}{(D-3)(D-2)} \\
& \times\left\{\overline{\mathcal{D}}_{\mu} \overline{\mathcal{D}}_{\nu} K_{A C}+2 \delta^{0}{ }_{(\mu} \overline{\mathcal{D}}_{\nu)} \mathcal{D}_{0} K_{B C}+\delta^{0}{ }_{\mu} \delta^{0}{ }_{\nu} \mathcal{D}_{0}{ }^{2} K_{C C}+\frac{\bar{\eta}_{\mu \nu} \mathcal{D}_{0}{ }^{2}\left(K_{C C}-K_{A C}\right)}{D-3}\right\}
\end{aligned}
$$

Unlike the $\delta \alpha$ perturbation (3.24), the $\delta \beta$ perturbation has only diagonal tensor factors and involves no $J_{\mu \nu}\left(x ; x^{\prime}\right)$ integrals.

\section{Discussion}

This paper concerns gauge dependence in the graviton propagator on de Sitter background. In section 2 we generalized the simple gauge condition (2.2) to a 2-parameter family of 
gauges (2.7). The flat space limit of this family coincides with the gauges employed in a study of how source and observer corrections cancel gauge dependence in the effective field equation [16]. In section 3 we argued that just the first order perturbations around the simple gauge provide two thirds of the checks made in that study. Our results for these first order perturbations are equations (3.24) and (3.26). They are expressed in terms of integrated propagators that are evaluated in the appendix. All our work was done in $D$ spacetime dimensions to facilitate the use of dimensional regularization.

Even the first order perturbations are very complicated, and we do not advocate using them for routine calculations. Their purpose is to provide an explicit check that our technique for canceling gauge dependence [16] works on de Sitter background. Establishing that fact is crucial, but once it has been done, we expect that future computations will be made using the simple gauge propagator $[11,12]$. The advantages of this propagator are that its $D=4$ limit is simple, that its tensor factors are independent of space and time, and that the 1PI 2-point functions it produces are elementary functions of the scale factors and the conformal coordinate interval. This is why just one loop computation has been done in another gauge, and that computation was only made to check gauge dependence.

The chief source of complication in our analysis is the factors of $1 / a$ and $1 / a^{2}$ that accompany derivatives inside convolutions of propagators (3.12)-(3.13). This becomes apparent in the appendix, when comparing the trivial result (A.10)-(A.11) for the $I_{\mu \nu}\left(x ; x^{\prime}\right)$ convolution, which contains no factors of $1 / a$, with the terrific effort expended to determine the $J_{\mu \nu}\left(x ; x^{\prime}\right)$ and $K_{\mu \nu}\left(x ; x^{\prime}\right)$ convolutions. In addition to facilitating checks of gauge dependence, this work should prove useful for computing the expectation values of gauge invariant measures of back-reaction [25]. Were we to push one order higher in the perturbations $\delta \alpha$ and $\delta \beta$ there would be another convolution, but no more factors of $1 / a$ within any one integration, so perhaps the extra labor would not be prohibitive. Note that including even one quadratic perturbation in flat space would suffice to cover the full range of checks available in table 1 from the all-orders result. Further, only two convolutions are required for the full propagator (3.1) in flat space, so perhaps an all-orders result could be obtained as well in de Sitter.

Finally, we should lay out our program of research concerning gauge dependence on de Sitter background:

1. Use the one graviton loop contribution to the 1PI 2-point function of a massless, minimally coupled scalar [3] to check for logarithmic corrections to the mode functions and the scalar exchange potential.

2. Use the simple propagator to include source and observer corrections to see how large logarithms are affected.

3. Re-do 1-2 using (3.24) and (3.26) to check that source and observer corrections cancel gauge dependence.

\section{Acknowledgments}

This work was partially supported by the Fonds de la Recherche Scientifique — FNRS under Grant IISN 4.4517.08 — Theory of fundamental interactions, by Taiwan MOST 
grants 103-2112-M-006-001-MY3 and 107-2119-M-006-014; by the D-ITP consortium, a program of the Netherlands Organization for Scientific Research (NWO) that is funded by the Dutch Ministry of Education, Culture and Science (OCW); by NSF grants PHY1806218 and PHY-1912484; and by the Institute for Fundamental Theory at the University of Florida.

\section{A Integrated propagators}

This appendix concerns technical details of the three integrated propagators (3.11)-(3.13). We begin by explaining how to reflect derivatives from one argument of a propagator to the other. We then give exact results for $I_{\mu \nu}\left(x ; x^{\prime}\right)$, and derive expansions for $J_{\mu \nu}\left(x ; x^{\prime}\right)$ and $K_{\mu \nu}\left(x ; x^{\prime}\right)$.

\section{A.1 Reflecting derivatives}

Although we choose to keep the derivatives of (3.14)-(3.16) on the dummy variable $z^{\mu}$, they could be reflected to the external variables using some identities which were originally derived in [26]. Of course space derivatives reflect the same as on flat space background,

$$
\partial_{i} i \Delta_{\nu}\left(x ; x^{\prime}\right)=-\partial_{i}^{\prime} i \Delta_{\nu}\left(x ; x^{\prime}\right) .
$$

Reflecting the time derivatives requires that we explain the relation between the index $\nu$ in the scalar propagator $i \Delta_{\nu}\left(x ; x^{\prime}\right)$ and the scalar mass $m$,

$$
\nu=\sqrt{\frac{(D-1)^{2}}{4}-\frac{m^{2}}{H^{2}}} .
$$

The three scalar propagators we employ correspond to masses and indices,

$$
\begin{array}{ll}
A \Longrightarrow m_{A}^{2}=0, & \nu_{A}=\left(\frac{D-1}{2}\right), \\
B \Longrightarrow m_{B}^{2}=(D-2) H^{2}, & \nu_{B}=\left(\frac{D-3}{2}\right)=\nu_{A}-1, \\
C \Longrightarrow m_{C}^{2}=2(D-3) H^{2}, & \nu_{C}=\left(\frac{D-5}{2}\right)=\nu_{B}-1 .
\end{array}
$$

The temporal reflection identities are,

$$
\begin{aligned}
& {\left[\partial_{0}+\left(\nu_{A}-\nu\right) H a\right] i \Delta_{\nu}\left(x ; x^{\prime}\right)=-\left[\partial_{0}^{\prime}+\left(\nu_{A}+\nu-1\right) H a^{\prime}\right] i \Delta_{\nu-1}\left(x ; x^{\prime}\right),} \\
& {\left[\partial_{0}+\left(\nu_{A}+\nu\right) H a\right] i \Delta_{\nu}\left(x ; x^{\prime}\right)=-\left[\partial_{0}^{\prime}+\left(\nu_{A}-\nu-1\right) H a^{\prime}\right] i \Delta_{\nu+1}\left(x ; x^{\prime}\right) .}
\end{aligned}
$$

The specific reflection identities we need are,

$$
\begin{aligned}
\partial_{0} i \Delta_{A}\left(x ; x^{\prime}\right) & =-\left[\partial_{0}^{\prime}+(D-2) H a^{\prime}\right] i \Delta_{B}\left(x ; x^{\prime}\right), \\
\left(\partial_{0}+H a\right) i \Delta_{B}\left(x ; x^{\prime}\right) & =-\left[\partial_{0}^{\prime}+(D-3) H a^{\prime}\right] i \Delta_{C}\left(x ; x^{\prime}\right) .
\end{aligned}
$$




\section{A.2 Results}

Exact results can be derived for $I_{\mu \nu}\left(x ; x^{\prime}\right)[27]$,

$$
\begin{aligned}
& I_{\mu \nu}\left(x ; x^{\prime}\right)=\frac{i \Delta_{\mu}\left(x ; x^{\prime}\right)-i \Delta_{\nu}\left(x ; x^{\prime}\right)}{m_{\mu}^{2}-m_{\nu}^{2}}, \quad \mu \neq \nu, \\
& I_{\mu \mu}\left(x ; x^{\prime}\right)=\frac{-1}{2 \mu H^{2}} \frac{\partial}{\partial \mu} i \Delta_{\mu}\left(x ; x^{\prime}\right),
\end{aligned}
$$

where from now on we use a shorthand notation $\nu_{\mu} \equiv \mu$. Although an asymptotic expansion can be derived for $J_{\mu \nu}\left(x ; x^{\prime}\right)$ [25], it is better, for our purposes, to use the reflection identities (A.8) and (A.9) to express all the $J$-propagators in terms of derivatives of the $K$ propagators,

$$
\begin{aligned}
& J_{A A}=\frac{1}{(D-2) H}\left\{\left[\partial_{0}+(D-2) H a\right] K_{B A}+\left[\partial_{0}^{\prime}+(D-2) H a^{\prime}\right] K_{A B}\right\}, \\
& J_{B B}=\frac{-1}{(D-2) H}\left\{\partial_{0} K_{A B}+\partial_{0}^{\prime} K_{B A}\right\}, \\
& J_{C C}=\frac{-1}{(D-4) H}\left\{\left[\partial_{0}+H a\right] K_{B C}+\left[\partial_{0}^{\prime}+H a^{\prime}\right] K_{C B}\right\}, \\
& J_{A B}=\frac{1}{(D-3) H}\left\{\left[\partial_{0}+(D-2) H a\right] K_{B B}+\left[\partial_{0}^{\prime}+(D-3) H a^{\prime}\right] K_{A C}\right\}, \\
& J_{A C}=\frac{1}{H}\left\{\left[\partial_{0}+(D-2) H a\right] K_{B C}+\left[\partial_{0}^{\prime}+H a^{\prime}\right] K_{A B}\right\}, \\
& J_{B C}=\frac{-1}{(D-3) H}\left\{\partial_{0} K_{A C}+\left[\partial_{0}^{\prime}+H a^{\prime}\right] K_{B B}\right\} .
\end{aligned}
$$

Thus we only need to compute the simpler $K$-propagators, which we do in the remainder of the appendix. If necessary, analogous relations expressing $I$-propagators in terms of the derivatives of the $J$-propagators can be constructed and used to check the correctness of the solutions.

We determine the $K$-propagators by solving the two equations of motion they satisfy,

$$
\begin{aligned}
& \left(\square-M_{\mu}^{2}\right) K_{\mu \nu}\left(x ; x^{\prime}\right)=\frac{1}{a^{2}} \times i \Delta_{\nu}\left(x ; x^{\prime}\right), \\
& \left(\square^{\prime}-M_{\nu}^{2}\right) K_{\mu \nu}\left(x ; x^{\prime}\right)=\frac{1}{a^{2}} \times i \Delta_{\mu}\left(x ; x^{\prime}\right)
\end{aligned}
$$

Instead of the two coordinates $x$ and $x^{\prime}$, it is convenient to use different variables - the de Sitter invariant distance $y$ defined in (3.7), and the two-time variables $u=\ln \left(a a^{\prime}\right)$ and $v=\ln \left(a / a^{\prime}\right)$. Furthermore, it is convenient to rescale the $K$-propagators,

$$
K_{\mu \nu}\left(x ; x^{\prime}\right)=\frac{H^{D-2} \Gamma\left(\frac{D-2}{2}\right)}{(4 \pi)^{D / 2}} \times \frac{e^{-u}}{H^{2}} \times \mathcal{K}_{\mu \nu}(y, u, v)
$$


and to consider the difference and the sum of the equations (A.18) and (A.19), respectively,

$$
\begin{gathered}
\left\{8 \frac{\partial}{\partial v}\left[\operatorname{sh}^{2}\left(\frac{v}{2}\right) \frac{\partial}{\partial y}\right]-4 \operatorname{sh}(v) \frac{\partial}{\partial u} \frac{\partial}{\partial y}\right. \\
\left.+\left[2 y \frac{\partial}{\partial y}+2 \frac{\partial}{\partial u}+D-3\right] \frac{\partial}{\partial v}-\frac{\left(\mu^{2}-\nu^{2}\right)}{2}\right\} \mathcal{K}_{\mu \nu}(y, u, v) \\
=\operatorname{sh}(v) i \Delta_{\mu \nu}^{+}(y, u)+\operatorname{ch}(v) i \Delta_{\mu \nu}^{-}(y, u), \\
\left\{\left[\left(4 y-y^{2}\right) \frac{\partial}{\partial y}-\left(D-2+2 \frac{\partial}{\partial u}\right) y-8 \operatorname{sh}^{2}\left(\frac{v}{2}\right) \frac{\partial}{\partial u}+2(D-2)\right] \frac{\partial}{\partial y}\right. \\
-\frac{\partial^{2}}{\partial v^{2}}+4 \frac{\partial}{\partial v}\left[\operatorname{sh}(v) \frac{\partial}{\partial y}\right]-\frac{\partial^{2}}{\partial u^{2}}-(D-3) \frac{\partial}{\partial u} \\
\left.+\frac{\left(\mu^{2}+\nu^{2}\right)}{2}-\frac{(D-3)^{2}}{4}\right\} \mathcal{K}_{\mu \nu}(y, u, v) \\
=\operatorname{ch}(v) i \Delta_{\mu \nu}^{+}(y, u)+\operatorname{sh}(v) i \Delta_{\mu \nu}^{-}(y, u),
\end{gathered}
$$

where the sources on the right hand side are rescaled sums and differences of the scalar propagators (3.8)-(3.10), defined as,

$$
i \Delta_{\mu \nu}^{ \pm}(y, u)=\left[\frac{H^{D-2} \Gamma\left(\frac{D-2}{2}\right)}{(4 \pi)^{D / 2}}\right]^{-1} \times \frac{1}{2}\left[i \Delta_{\mu}\left(x ; x^{\prime}\right) \pm i \Delta_{\nu}\left(x ; x^{\prime}\right)\right] .
$$

We solve the equations (A.21) and (A.22) as a power series in $y$ around $y=0$. In order to satisfy the original equations (A.18) and (A.19), this power series has to take the form,

$$
\begin{aligned}
\mathcal{K}_{\mu \nu}(y, u, v)= & -\frac{2\left(E_{\mu \nu}\right)_{0}}{(D-4)}\left(\frac{y}{4}\right)^{-\frac{D-4}{2}}+\frac{2\left(F_{\mu \nu}\right)_{0}}{(D-4)} \\
& +\sum_{n=1}^{\infty}\left[\frac{\left(E_{\mu \nu}\right)_{n}}{n-\frac{D-4}{2}}\left(\frac{y}{4}\right)^{n-\frac{D-4}{2}}-\frac{\left(F_{\mu \nu}\right)_{n}}{n}\left(\frac{y}{4}\right)^{n}\right],
\end{aligned}
$$

where numerical factors have been taken out for convenience, and where the coefficients $\left(E_{\mu \nu}\right)_{n}$ and $\left(F_{\mu \nu}\right)_{n}$ that we need to determine are functions of variables $u$ and $v$ in general. The equations of motion for these coefficients follow from the full equations (A.21) and (A.22). It is useful to define an analogous power series expansion of the sum and the difference of the scalar propagators from (A.23),

$$
\begin{aligned}
& i \Delta_{\mu \nu}^{+}\left(x ; x^{\prime}\right)=\left(\frac{y}{4}\right)^{-\frac{D-2}{2}}+\sum_{n=0}^{\infty}\left[\left(S_{\mu \nu}^{+}\right)_{n}\left(\frac{y}{4}\right)^{n-\frac{D-4}{2}}-\left(Q_{\mu \nu}^{+}\right)_{n}\left(\frac{y}{4}\right)^{n}\right] \\
& i \Delta_{\mu \nu}^{-}\left(x ; x^{\prime}\right)=\sum_{n=0}^{\infty}\left[\left(S_{\mu \nu}^{-}\right)_{n}\left(\frac{y}{4}\right)^{n-\frac{D-4}{2}}-\left(Q_{\mu \nu}^{-}\right)_{n}\left(\frac{y}{4}\right)^{n}\right]
\end{aligned}
$$


where the coefficients of these expansions can be read off by comparing (A.23) to the power series of the scalar propagators (3.8)-(3.10),

$$
\begin{aligned}
& \left(S_{\mu \nu}^{ \pm}\right)_{n}=\frac{\Gamma\left(\frac{4-D}{2}\right)}{2(n+1) ! \Gamma\left(\frac{6-D}{2}+n\right)} \\
& (n \geq 0) \\
& \times\left[\frac{\Gamma\left(\frac{3}{2}+\mu+n\right) \Gamma\left(\frac{3}{2}-\mu+n\right)}{\Gamma\left(\frac{1}{2}+\mu\right) \Gamma\left(\frac{1}{2}-\mu\right)} \pm \frac{\Gamma\left(\frac{3}{2}+\nu+n\right) \Gamma\left(\frac{3}{2}-\nu+n\right)}{\Gamma\left(\frac{1}{2}+\nu\right) \Gamma\left(\frac{1}{2}-\nu\right)}\right], \\
& \left(Q_{A A}^{ \pm}\right)_{0}=\frac{\Gamma(D-1)}{2 \Gamma\left(\frac{D}{2}\right) \Gamma\left(\frac{D-2}{2}\right)}\left[-\frac{A_{1} \Gamma\left(\frac{D}{2}\right)}{\Gamma(D-1)}-u\right](1 \pm 1), \\
& \left(Q_{B B}^{ \pm}\right)_{0}=\frac{\Gamma(D-2)}{2 \Gamma\left(\frac{D}{2}\right) \Gamma\left(\frac{D-2}{2}\right)}(1 \pm 1), \\
& \left(Q_{C C}^{ \pm}\right)_{0}=-\frac{\Gamma(D-3)}{2 \Gamma\left(\frac{D}{2}\right) \Gamma\left(\frac{D-2}{2}\right)}(1 \pm 1), \\
& \left(Q_{A B}^{ \pm}\right)_{0}=\frac{\Gamma(D-1)}{2 \Gamma\left(\frac{D}{2}\right) \Gamma\left(\frac{D-2}{2}\right)}\left[-\frac{A_{1} \Gamma\left(\frac{D}{2}\right)}{\Gamma(D-1)} \pm \frac{1}{(D-2)}-u\right], \\
& \left(Q_{A C}^{ \pm}\right)_{0}=\frac{\Gamma(D-1)}{2 \Gamma\left(\frac{D}{2}\right) \Gamma\left(\frac{D-2}{2}\right)}\left[-\frac{A_{1} \Gamma\left(\frac{D}{2}\right)}{\Gamma(D-1)} \mp \frac{1}{(D-2)(D-3)}-u\right], \\
& \left(Q_{B C}^{ \pm}\right)_{0}=\frac{\Gamma(D-3)}{2 \Gamma\left(\frac{D}{2}\right) \Gamma\left(\frac{D-2}{2}\right)}(D-3 \mp 1) \\
& \left(Q_{\mu \nu}^{ \pm}\right)_{n}=\frac{\Gamma\left(\frac{4-D}{2}\right)}{2 n ! \Gamma\left(\frac{D}{2}+n\right)} \\
& \times\left[\frac{\Gamma\left(\frac{D-1}{2}+n+\mu\right) \Gamma\left(\frac{D-1}{2}+n-\mu\right)}{\Gamma\left(\frac{1}{2}+\mu\right) \Gamma\left(\frac{1}{2}-\mu\right)} \pm \frac{\Gamma\left(\frac{D-1}{2}+n+\nu\right) \Gamma\left(\frac{D-1}{2}+n-\nu\right)}{\Gamma\left(\frac{1}{2}+\nu\right) \Gamma\left(\frac{1}{2}-\nu\right)}\right],
\end{aligned}
$$

where the constant $A_{1}$ is,

$$
A_{1}=-\frac{\Gamma(D-1)}{\Gamma\left(\frac{D}{2}\right)} \pi \cot \left(\pi \frac{D}{2}\right)
$$

and where $\psi(z)=\Gamma^{\prime}(z) / \Gamma(z)$ is the digamma function. Note that some coefficients from the integer series depend linearly on $u=\ln \left(a a^{\prime}\right)$, and that this dependence descends from the de Sitter breaking part of the $A$-type propagator (3.8).

Since the equations for the coefficients of the non-integer power series (A.24) decouple from the equations for the integer ones, we solve for them separately in the following subsections. 


\section{A.2.1 Non-integer power series}

The equations for the non-integer power series coefficients, descending from eq. (A.21) are,

$$
\begin{array}{cc}
2 \frac{\partial}{\partial v}\left[\operatorname{sh}^{2}\left(\frac{v}{2}\right)\left(E_{\mu \nu}\right)_{0}\right]-\operatorname{sh}(v) \frac{\partial}{\partial u}\left(E_{\mu \nu}\right)_{0}=\operatorname{sh}(v), & (n \geq 1) \\
2 \frac{\partial}{\partial v}\left[\operatorname{sh}^{2}\left(\frac{v}{2}\right)\left(E_{\mu \nu}\right)_{n}\right]-\operatorname{sh}(v) \frac{\partial}{\partial u}\left(E_{\mu \nu}\right)_{n} & \quad\left[\frac{-1}{\left(n-\frac{D-2}{2}\right)}\left[\left(2 n-1+2 \frac{\partial}{\partial u}\right) \frac{\partial}{\partial v}-\frac{\left(\mu^{2}-\nu^{2}\right)}{2}\right]\left(E_{\mu \nu}\right)_{n-1}\right. \\
+\operatorname{sh}(v)\left(S_{\mu \nu}^{+}\right)_{n-1}+\operatorname{ch}(v)\left(S_{\mu \nu}^{-}\right)_{n-1} .
\end{array}
$$

while the ones descending from eq. (A.22) are,

$$
\begin{gathered}
\frac{\partial}{\partial v}\left[\operatorname{sh}(v)\left(E_{\mu \nu}\right)_{0}\right]-2 \operatorname{sh}^{2}\left(\frac{v}{2}\right) \frac{\partial}{\partial u}\left(E_{\mu \nu}\right)_{0}=\operatorname{ch}(v), \\
\frac{\partial}{\partial v}\left[\operatorname{sh}(v)\left(E_{\mu \nu}\right)_{n}\right]-2 \operatorname{sh}^{2}\left(\frac{v}{2}\right) \frac{\partial}{\partial u}\left(E_{\mu \nu}\right)_{n}+n\left(E_{\mu \nu}\right)_{n} \quad(n \geq 1) \\
=\frac{1}{\left(n-\frac{D-2}{2}\right)}\left[\left(n-\frac{D-2}{2}\right)\left(n+\frac{D-4}{2}+2 \frac{\partial}{\partial u}\right)+\frac{\partial^{2}}{\partial v^{2}}\right. \\
\left.+\frac{\partial^{2}}{\partial u^{2}}+(D-3) \frac{\partial}{\partial u}+\frac{(D-3)^{2}}{4}-\frac{\left(\mu^{2}+\nu^{2}\right)}{2}\right]\left(E_{\mu \nu}\right)_{n-1} \\
+\operatorname{ch}(v)\left(S_{\mu \nu}^{+}\right)_{n-1}+\operatorname{sh}(v)\left(S_{\mu \nu}^{-}\right)_{n-1} .
\end{gathered}
$$

The leading coefficient that simultaneously solves (A.36) and (A.38), and is finite in the time coincidence limit (i.e. limit $v \rightarrow 0$ ) is unique,

$$
\left(E_{\mu \nu}\right)_{0}=1
$$

and is independent of the indices $(\mu, \nu=A, B, C)$. This solution is taken as the germ of the recurrence in (A.37), which can be easily integrated as it is only a first order differential equation. Iterating this equation generates higher coefficients of the expansion, and all the integration constants are uniquely fixed by demanding expressions to be finite for $v \rightarrow 0$. The first couple of higher order coefficients are,

$$
\begin{aligned}
&\left(E_{\mu \nu}\right)_{1}=\left(S_{\mu \nu}^{+}\right)_{0}+\frac{\left(\mu^{2}-\nu^{2}\right)}{2(D-4)}\left[\frac{\operatorname{sh}(v)-v}{\operatorname{sh}^{2}\left(\frac{v}{2}\right)}\right] \\
&\left(E_{\mu \nu}\right)_{2}=\left(S_{\mu \nu}^{+}\right)_{1}+\frac{3\left(\mu^{2}-\nu^{2}\right)}{2(D-4)(D-6)}\left[\frac{3 \operatorname{sh}(v)-v[1+2 \operatorname{ch}(v)]}{3 \operatorname{sh}^{4}\left(\frac{v}{2}\right)}\right] \\
&+\frac{\left(\mu^{2}-\nu^{2}\right)}{2(D-4)(D-6)}\left[\frac{2 \operatorname{sh}\left(\frac{v}{2}\right)-v \operatorname{ch}\left(\frac{v}{2}\right)}{\operatorname{sh}^{3}\left(\frac{v}{2}\right)}\right] \\
& \quad+\frac{\left(\mu^{2}-\nu^{2}\right)\left(\mu^{2}+\nu^{2}-\frac{5}{2}\right)}{2(D-4)(D-6)}\left[\frac{\operatorname{sh}(v)-v}{\operatorname{sh}^{2}\left(\frac{v}{2}\right)}\right]
\end{aligned}
$$


Specialization of the expressions above to particular types $A, B, C$ is simply accomplished by plugging in the specific parameters (A.3)-(A.5). As opposed to the general case where the coefficients take complicated forms above, in the special case of diagonal propagators $(\nu=\mu)$ we can solve the recurrence in a closed form to all orders,

$$
\left(E_{\mu \mu}\right)_{0}=1, \quad\left(E_{\mu \mu}\right)_{n}=\left(S_{\mu \mu}^{+}\right)_{n-1}, \quad(n \geq 1) .
$$

\section{A.2.2 Integer power series}

The equations for the coefficients of the integer power series in (A.24) that descend from (A.21) are,

$$
\begin{aligned}
& 2 \frac{\partial}{\partial v}\left[\operatorname{sh}^{2}\left(\frac{v}{2}\right)\left(F_{\mu \nu}\right)_{1}\right]-\operatorname{sh}(v) \frac{\partial}{\partial u}\left(F_{\mu \nu}\right)_{1} \\
& -\frac{2}{(D-4)}\left[\left(2 \frac{\partial}{\partial u}+D-3\right) \frac{\partial}{\partial v}-\frac{\left(\mu^{2}-\nu^{2}\right)}{2}\right]\left(F_{\mu \nu}\right)_{0} \\
& \quad=\operatorname{sh}(v)\left(Q_{\mu \nu}^{+}\right)_{0}+\operatorname{ch}(v)\left(Q_{\mu \nu}^{-}\right)_{0} \\
& 2 \frac{\partial}{\partial v}\left[\operatorname{sh}^{2}\left(\frac{v}{2}\right)\left(F_{\mu \nu}\right)_{n}\right]-\operatorname{sh}(v) \frac{\partial}{\partial u}\left(F_{\mu \nu}\right)_{n} \\
& \quad=\frac{-1}{(n-1)}\left[\left(2 n+D-5+2 \frac{\partial}{\partial u}\right)_{\frac{\partial}{\partial v}}-\frac{\left(\mu^{2}-\nu^{2}\right)}{2}\right]\left(F_{\mu \nu}\right)_{n-1} \\
& \quad+\operatorname{sh}(v)\left(Q_{\mu \nu}^{+}\right)_{n-1}+\operatorname{ch}(v)\left(Q_{\mu \nu}^{-}\right)_{n-1}
\end{aligned}
$$

and the equations that descend from (A.22),

$$
\begin{gathered}
\frac{\partial}{\partial v}\left[\operatorname{sh}(v)\left(F_{\mu \nu}\right)_{1}\right]+\left[\frac{D-2}{2}-2 \operatorname{sh}^{2}\left(\frac{v}{2}\right) \frac{\partial}{\partial u}\right]\left(F_{\mu \nu}\right)_{1} \\
+\frac{2}{(D-4)}\left[\frac{\partial^{2}}{\partial v^{2}}+\frac{\partial^{2}}{\partial u^{2}}+(D-3) \frac{\partial}{\partial u}+\frac{(D-3)^{2}}{4}-\frac{\left(\mu^{2}+\nu^{2}\right)}{2}\right]\left(F_{\mu \nu}\right)_{0} \\
=\operatorname{ch}(v)\left(Q_{\mu \nu}^{+}\right)_{0}+\operatorname{sh}(v)\left(Q_{\mu \nu}^{-}\right)_{0}, \quad(n \geq 2) \\
\frac{\partial}{\partial v}\left[\operatorname{sh}(v)\left(F_{\mu \nu}\right)_{n}\right]+\left[n+\frac{D-4}{2}-2 \operatorname{sh}^{2}\left(\frac{v}{2}\right) \frac{\partial}{\partial u}\right]\left(F_{\mu \nu}\right)_{n} \quad(2 n+D-5) \frac{\partial}{\partial u} \\
=\frac{1}{(n-1)}\left[\frac{\partial^{2}}{\partial v^{2}}+\frac{\partial^{2}}{\partial u^{2}}+(2 n-3)^{2}\right. \\
+\left(n-\frac{\left(\mu^{2}+\nu^{2}\right)}{2}\right]\left(F_{\mu \nu}\right)_{n-1} \\
+\operatorname{ch}(v)\left(Q_{\mu \nu}^{+}\right)_{n-1}+\operatorname{sh}(v)\left(Q_{\mu \nu}^{-}\right)_{n-1} .
\end{gathered}
$$

In the equations above the first two leading coefficients satisfy two coupled equations (A.44) and (A.46), as opposed to the equations for non-integer coefficients where only the leading 
coefficient appears in the leading order equations. This difference complicates the problem since now the first two leading coefficients are required to set off the recurrence defining the higher order coefficients.

For the diagonal propagators we can find the coefficients at all orders in a closed form,

$$
\begin{array}{ll}
\left(F_{A A}\right)_{0}=\frac{(D-4)}{4}\left(Q_{A A}^{+}\right)_{0}-\frac{(2 D-3) \Gamma(D-2)}{2 \Gamma\left(\frac{D}{2}\right) \Gamma\left(\frac{D-4}{2}\right)}, & \\
\left(F_{A A}\right)_{1}=\left(Q_{A A}^{+}\right)_{0}-\frac{\Gamma(D-1)}{\Gamma\left(\frac{D}{2}\right) \Gamma\left(\frac{D-2}{2}\right)}, & (n \geq 2) \\
\left(F_{A A}\right)_{n}=\left(Q_{A A}^{+}\right)_{n-1}, & (n \geq 1) \\
\left(F_{B B}\right)_{0}=1-\frac{(D-2)(D-4)}{4(D-3)}\left(Q_{B B}^{+}\right)_{0} u, & \\
\left(F_{B B}\right)_{n}=\left(Q_{B B}^{+}\right)_{n-1}, & (n \geq 1) \\
\left(F_{C C}\right)_{0}=-\frac{(D-2)}{4}\left(Q_{C C}^{+}\right)_{0}+\frac{1}{2} e^{-(D-4) u}, & \\
\left(F_{C C}\right)_{n}=\left(Q_{C C}^{+}\right)_{n-1} . &
\end{array}
$$

The homogeneous parts of the solutions for the leading coefficients were fixed by requiring that the limit $D \rightarrow 4$ of the full propagator exists off-coincidence.

The off-diagonal coefficients are considerably more difficult to solve for, and we give the solutions for the coefficients in terms of the power series in $v$,

$$
\left(F_{\mu \nu}\right)_{n}=\sum_{k=0}^{\infty}\left(F_{\mu \nu}\right)_{n}^{k} v^{k}
$$

This expansion, together with power series in $y$ from (A.24), makes the answer for the off-diagonal $K$-propagators take the form of a double power series in terms less and less singular in the space-time coincidence limit. The solutions for the coefficients in (A.55) are found by expanding the hyperbolic functions in eqs. (A.44)-(A.45), and organizing the equations by orders in $v$. The two leading equations (A.44) and (A.46) have to be solved first, as they couple only $\left(F_{\mu \nu}\right)_{0}$ and $\left(F_{\mu \nu}\right)_{1}$. We give the first several terms in the $v$-expansion of these two in the following form,

$$
\begin{aligned}
\left(F_{\mu \nu}\right)_{0}^{0}= & \frac{(D-4)}{\left(\mu^{2}-\nu^{2}\right)}\left(Q_{\mu \nu}^{-}\right)_{0}, \quad\left(F_{\mu \nu}\right)_{0}^{1}=0 \\
\left(F_{\mu \nu}\right)_{0}^{2}= & \frac{-D(D-4)}{4(D-1)(D-2)}\left[1-\frac{2}{(D-2)} \frac{\partial}{\partial u}\right]\left\{\frac{1}{D}\left[D-2+2 \frac{\partial}{\partial u}\right]\left(Q_{\mu \nu}^{+}\right)_{0}\right. \\
& \left.+\left(1+\frac{\partial}{\partial u}\right)\left[(D-3) \frac{\partial}{\partial u}+\frac{(D-3)^{2}}{4}-\frac{\left(\mu^{2}+\nu^{2}\right)}{2}\right] \frac{4\left(Q_{\mu \nu}^{-}\right)_{0}}{D\left(\mu^{2}-\nu^{2}\right)}\right\} \\
\left(F_{\mu \nu}\right)_{1}^{0}= & \frac{2}{D}\left\{-\frac{4\left(F_{\mu \nu}\right)_{0}^{2}}{(D-4)}+\left(Q_{\mu \nu}^{+}\right)_{0}\right. \\
& \left.\quad-\left[(D-3) \frac{\partial}{\partial u}+\frac{(D-3)^{2}}{4}-\frac{\left(\mu^{2}+\nu^{2}\right)}{2}\right] \frac{2\left(Q_{\mu \nu}^{-}\right)_{0}}{\left(\mu^{2}-\nu^{2}\right)}\right\}
\end{aligned}
$$




$$
\begin{aligned}
&\left(F_{\mu \nu}\right)_{0}^{3}=\frac{-(D+2)(D-4)}{6 D(D-1)}\left[1-\frac{2}{(D-1)} \frac{\partial}{\partial u}\right] \\
& \quad \times\left\{\frac{1}{2}\left(Q_{\mu \nu}^{-}\right)_{0}-\frac{\left(\mu^{2}-\nu^{2}\right)}{(D-4)}\left(F_{\mu \nu}\right)_{0}^{2}-\left(\frac{3}{2}-\frac{\partial}{\partial u}\right) \frac{2\left(Q_{\mu \nu}^{-}\right)_{0}}{(D+2)}\right\}, \\
&\left(F_{\mu \nu}\right)_{1}^{1}=\frac{2}{(D+2)}\left[\left(Q_{\mu \nu}^{-}\right)_{0}-\frac{12}{(D-4)}\left(F_{\mu \nu}\right)_{0}^{3}\right],
\end{aligned}
$$

which is also the order in which they are conveniently solved for. In the expressions above all the coefficients are assumed to be (at most) linear functions of $u$, as is also true for the coefficients (A.31)-(A.34). The leading coefficient in (A.56) and other homogeneous solutions are chosen such that (i) the regular $(D-4)$ limit of the full $K$-propagators exists, (ii) the subleading coefficients take simpler form. This choice is consistent due to the fact that the $\left(Q_{\mu \nu}^{ \pm}\right)$coefficients of the three types $(\mu, \nu)=(A, B, C)$ satisfy a consistency relation,

$$
\left[(D-1) \frac{\partial}{\partial u}+\frac{(D-1)^{2}}{4}-\frac{\left(\mu^{2}+\nu^{2}\right)}{2}\right]\left(Q_{\mu \nu}^{-}\right)_{0}+\frac{\left(\mu^{2}-\nu^{2}\right)}{2}\left(Q_{\mu \nu}^{+}\right)_{0}-\frac{D}{2}\left(Q_{\mu \nu}^{-}\right)_{1}=0,
$$

which guarantees that the solutions of the leading eqs. (A.44) and (A.46) that we found are consistent with higher order equations eqs. (A.45) and (A.47).

The higher order coefficients are now easily generated from the lower order ones (A.56)(A.60), by using either of equations (A.45) or (A.47), e.g.,

$$
\begin{aligned}
\left(F_{\mu \nu}\right)_{2}^{0}= & \frac{2}{(D+2)}\left\{2\left(F_{\mu \nu}\right)_{1}^{2}+\left(Q_{\mu \nu}^{+}\right)_{1}\right. \\
& \left.+\left[(D-1) \frac{\partial}{\partial u}+\frac{(D-1)^{2}}{4}-\frac{\left(\mu^{2}+\nu^{2}\right)}{2}\right]\left(F_{\mu \nu}\right)_{1}^{0}\right\}, \\
\left(F_{\mu \nu}\right)_{2}^{1}= & -\frac{2}{(D+1)}\left[1+\frac{2}{(D+1)} \frac{\partial}{\partial u}\right]\left\{\frac{\left(\mu^{2}-\nu^{2}\right)}{4}\left(F_{\mu \nu}\right)_{2}^{0}+\left(Q_{\mu \nu}^{-}\right)_{2}\right\}, \\
\left(F_{\mu \nu}\right)_{2}^{2}= & \frac{1}{(D+3)(D+2)}\left[1-\frac{2}{(D+2)} \frac{\partial}{\partial u}\right]\left\{\frac{(D+4)\left(\mu^{2}-\nu^{2}\right)}{4}\left(F_{\mu \nu}\right)_{2}^{1}\right. \\
& \left.-\left(1-\frac{\partial}{\partial u}\right)\left[(D+1) \frac{\partial}{\partial u}+\frac{(D+1)^{2}}{4}-\frac{\left(\mu^{2}+\nu^{2}\right)}{2}\right]\left(F_{\mu \nu}\right)_{2}^{0}\right\}+\frac{\left(Q_{\mu \nu}^{+}\right)_{2}}{(D+3)} .
\end{aligned}
$$

\section{A.2.3 Final expressions}

Having solved for all the coefficients of the diagonal $K$-propagators, we can write the full solutions in a closed form,

$$
\begin{aligned}
K_{A A}\left(x ; x^{\prime}\right)=\frac{e^{-u}}{H^{2}}\left\{\frac{1}{4} I[\right. & A(y)]+\frac{H^{D-2} \Gamma\left(\frac{D-2}{2}\right)}{(4 \pi)^{D / 2}} \times \frac{\Gamma(D-1)}{4 \Gamma\left(\frac{D}{2}\right) \Gamma\left(\frac{D-2}{2}\right)} \\
\times & {\left.\left[-\frac{2 A_{1} \Gamma\left(\frac{D}{2}\right)}{\Gamma(D-1)}-\frac{2(2 D-3)}{(D-2)}-(2-y) u+y\right]\right\}, }
\end{aligned}
$$




$$
\begin{aligned}
& K_{B B}\left(x ; x^{\prime}\right)=\frac{e^{-u}}{H^{2}}\left\{\frac{1}{4} I[B(y)]+\frac{H^{D-2} \Gamma\left(\frac{D-2}{2}\right)}{(4 \pi)^{D / 2}}\left[\frac{2}{(D-4)}-\frac{\Gamma(D-3)}{\Gamma^{2}\left(\frac{D-2}{2}\right)} u\right],\right. \\
& K_{C C}\left(x ; x^{\prime}\right)=\frac{e^{-u}}{H^{2}}\left\{\frac{1}{4} I[C(y)]+\frac{H^{D-2} \Gamma\left(\frac{D-2}{2}\right)}{(4 \pi)^{D / 2}}\left[\frac{\Gamma(D-4)}{\Gamma^{2}\left(\frac{D-2}{2}\right)}+\frac{e^{-(D-4) u}}{(D-4)}\right]\right\}
\end{aligned}
$$

where we define the primitive function $I[f(y)]=\int^{y} d y^{\prime} f\left(y^{\prime}\right)$. Four relations deriving from the reflection identities (A.8) and (A.9),

$$
\begin{aligned}
\partial_{0} K_{A A}+\left[\partial_{0}^{\prime}+(D-2) H a^{\prime}\right] K_{B B} & =0, \\
\partial_{0}^{\prime} K_{A A}+\left[\partial_{0}+(D-2) H a\right] K_{B B} & =0, \\
{\left[\partial_{0}+H a\right] K_{B B}+\left[\partial_{0}^{\prime}+(D-3) H a^{\prime}\right] K_{C C} } & =0, \\
{\left[\partial_{0}^{\prime}+H a^{\prime}\right] K_{B B}+\left[\partial_{0}+(D-3) H a\right] K_{C C} } & =0,
\end{aligned}
$$

can be used to check the consistency of the solutions.

The off-diagonal $K$-propagators can be given in terms of the power series (A.24), where the coefficients of the non-integer powers are given by (A.40)-(A.42) and the recurrence relation (A.37), and the leading coefficients of the integer powers are given in (A.56)-(A.60), with eqs. (A.44) and (A.47) generating the higher coefficients.

The expressions for the $J$-propagators are obtained from (A.12)-(A.17), by acting firstorder the derivative operators on the $K$-propagators whose solutions we have found here. A number of relations that relate the $J$-propagators with the $I$-propagators can be derived from reflection identities (A.8) and (A.9), e.g.

$$
\begin{aligned}
& \partial_{0} J_{A A}+\left[\partial_{0}^{\prime}+(D-2) H a^{\prime}\right] J_{B B}=H I_{A B}, \\
& \partial_{0}^{\prime} J_{A A}+\left[\partial_{0}+(D-2) H a\right] J_{B B}=H I_{A B} .
\end{aligned}
$$

and can be used to independently check the validity of the solutions.

Open Access. This article is distributed under the terms of the Creative Commons Attribution License (CC-BY 4.0), which permits any use, distribution and reproduction in any medium, provided the original author(s) and source are credited.

\section{References}

[1] N.C. Tsamis and R.P. Woodard, One loop graviton selfenergy in a locally de Sitter background, Phys. Rev. D 54 (1996) 2621 [hep-ph/9602317] [INSPIRE].

[2] S.P. Miao and R.P. Woodard, The Fermion self-energy during inflation, Class. Quant. Grav. 23 (2006) 1721 [gr-qc/0511140] [INSPIRE].

[3] E.O. Kahya and R.P. Woodard, Quantum Gravity Corrections to the One Loop Scalar Self-Mass during Inflation, Phys. Rev. D 76 (2007) 124005 [arXiv:0709.0536] [INSPIRE].

[4] S.P. Miao, Quantum Gravitational Effects on Massive Fermions during Inflation I, Phys. Rev. D 86 (2012) 104051 [arXiv:1207.5241] [INSPIRE]. 
[5] K.E. Leonard and R.P. Woodard, Graviton Corrections to Vacuum Polarization during Inflation, Class. Quant. Grav. 31 (2014) 015010 [arXiv:1304.7265] [INSPIRE].

[6] P.J. Mora, N.C. Tsamis and R.P. Woodard, Hartree approximation to the one loop quantum gravitationalcorrection to the graviton mode function on de Sitter, JCAP 10 (2013) 018 [arXiv: 1307.1422] [INSPIRE].

[7] S.P. Miao and R.P. Woodard, Gravitons Enhance Fermions during Inflation, Phys. Rev. D 74 (2006) 024021 [gr-qc/0603135] [INSPIRE].

[8] E.O. Kahya and R.P. Woodard, Scalar field equations from quantum gravity during inflation, Phys. Rev. D 77 (2008) 084012 [arXiv:0710.5282] [INSPIRE].

[9] C.L. Wang and R.P. Woodard, Excitation of Photons by Inflationary Gravitons, Phys. Rev. D 91 (2015) 124054 [arXiv: 1408.1448] [INSPIRE].

[10] D. Glavan, S.P. Miao, T. Prokopec and R.P. Woodard, Electrodynamic Effects of Inflationary Gravitons, Class. Quant. Grav. 31 (2014) 175002 [arXiv:1308.3453] [INSPIRE].

[11] N.C. Tsamis and R.P. Woodard, The Structure of perturbative quantum gravity on a de Sitter background, Commun. Math. Phys. 162 (1994) 217 [inSPIRE].

[12] R.P. Woodard, de Sitter breaking in field theory, in proceedings of the Deserfest: A Celebration of the Life and Works of Stanley Deser, Ann Arbor, Michigan, U.S.A., 3-5 April 2004, pp. 339-351 [gr-qc/0408002] [INSPIRE].

[13] P.J. Mora, N.C. Tsamis and R.P. Woodard, Graviton Propagator in a General Invariant Gauge on de Sitter, J. Math. Phys. 53 (2012) 122502 [arXiv:1205.4468] [INSPIRE].

[14] D. Glavan, S.P. Miao, T. Prokopec and R.P. Woodard, Graviton Loop Corrections to Vacuum Polarization in de Sitter in a General Covariant Gauge, Class. Quant. Grav. 32 (2015) 195014 [arXiv:1504.00894] [INSPIRE].

[15] D. Glavan, S.P. Miao, T. Prokopec and R.P. Woodard, One loop graviton corrections to dynamical photons in de Sitter, Class. Quant. Grav. 34 (2017) 085002 [arXiv:1609.00386] [INSPIRE].

[16] S.P. Miao, T. Prokopec and R.P. Woodard, Deducing Cosmological Observables from the S-matrix, Phys. Rev. D 96 (2017) 104029 [arXiv:1708.06239] [INSPIRE].

[17] J.F. Donoghue, Leading quantum correction to the Newtonian potential, Phys. Rev. Lett. 72 (1994) 2996 [gr-qc/9310024] [INSPIRE].

[18] J.F. Donoghue, General relativity as an effective field theory: The leading quantum corrections, Phys. Rev. D 50 (1994) 3874 [gr-qc/9405057] [INSPIRE].

[19] J.F. Donoghue and T. Torma, On the power counting of loop diagrams in general relativity, Phys. Rev. D 54 (1996) 4963 [hep-th/9602121] [INSPIRE].

[20] S.P. Miao, N.C. Tsamis and R.P. Woodard, Transforming to Lorentz Gauge on de Sitter, J. Math. Phys. 50 (2009) 122502 [arXiv:0907.4930] [INSPIRE].

[21] D.M. Capper, A General Gauge Graviton Loop Calculation, J. Phys. A 13 (1980) 199 [INSPIRE].

[22] K.E. Leonard and R.P. Woodard, Graviton Corrections to Maxwell's Equations, Phys. Rev. D 85 (2012) 104048 [arXiv:1202.5800] [INSPIRE].

[23] V.K. Onemli and R.P. Woodard, Superacceleration from massless, minimally coupled $\phi^{4}$, Class. Quant. Grav. 19 (2002) 4607 [gr-qc/0204065] [INSPIRE]. 
[24] V.K. Onemli and R.P. Woodard, Quantum effects can render $w<-1$ on cosmological scales, Phys. Rev. D 70 (2004) 107301 [gr-qc/0406098] [inSPIRE].

[25] S.P. Miao, N.C. Tsamis and R.P. Woodard, Invariant measure of the one-loop quantum gravitational backreaction on inflation, Phys. Rev. D 95 (2017) 125008 [arXiv: 1702.05694] [INSPIRE].

[26] N.C. Tsamis and R.P. Woodard, Mode analysis and ward identities for perturbative quantum gravity in de Sitter space, Phys. Lett. B 292 (1992) 269 [INSPIRE].

[27] S.P. Miao, N.C. Tsamis and R.P. Woodard, The Graviton Propagator in de Donder Gauge on de Sitter Background, J. Math. Phys. 52 (2011) 122301 [arXiv:1106.0925] [INSPIRE]. 